\title{
Old Disease and New Challenges: Major Obstacles of Current Strategies in the Prevention of Pertussis
}

\author{
Iraj Sedighi, ${ }^{1}$ Abdollah Karimi, ${ }^{2}$ and Ali Amanati ${ }^{3,}$ \\ ${ }^{1}$ Department of Pediatrics, Faculty of Medicine, Hamadan University of Medical Sciences, Hamadan, IR Iran \\ ${ }^{2}$ Pediatric Infections Research Center, Mofid Children's Hospital, Shahid Beheshti University of Medical Sciences, Tehran, IR Iran \\ ${ }^{3}$ Professor Alborzi Clinical Microbiology Research Center, Shiraz University of Medical Sciences, Shiraz, IR Iran \\ "Corresponding author: Ali Amanati, Professor Alborzi Clinical Microbiology Research Center, Shiraz University of Medical Sciences, Shiraz, IR Iran. E-mail: \\ ali_amanati_1356@yahoo.com
}

Received 2016 January 29; Revised 2016 April 26; Accepted 2016 May 23.

\begin{abstract}
Context: Universal immunization against Bordetella pertussis has partially controlled the burden of the disease and its transmission. However, according to recent data, the epidemiology of this vaccine-preventable disease has changed. Now, younger infants, adolescents, and adults are at greater risk of infection. This article has studied the interaction between the various factors involved in the changing epidemiology of pertussis and the major obstacles faced by the current strategies in its prevention.

Evidence Acquisition: In this narrative review, the most recently published sources of information on pertussis control measures, consisting of textbooks and articles, have been reviewed. We focused on the more recent data about the changing epidemiology or pertussis in Scopus through the use of the MeSH-term words [pertussis] or [whooping cough] and [epidemiology] or [outbreak] or [resurgence], but our search was not restricted to this particular strategy; we also tried to find all of the most recent available data in the general field through other means.

Results: Primary and booster doses of the pertussis vaccine seem to partially control transmission of the disease, but despite the different preventive strategies available, pertussis continues to cause mortality and morbidity among high-risk groups.

Conclusions: Adding booster doses of acellular pertussis vaccine to the current national immunization practices with whole-cell vaccines for young adults and pregnant women seems to be a good option for controlling mortality and morbidity among high-risk groups such as very young infants.
\end{abstract}

Keywords: Bordetella pertussis, Prevention and Control, Immunization, Pertussis Vaccine

\section{Context}

\subsection{Preface}

To better understand the epidemiology of pertussis, physicians must be familiar with basic epidemiologic concepts such as vaccine effectiveness (VE) and efficacy, effective transmission (or contact) rates, pathogen adaptation, pathogen fitness, and selection pressure. Because discussion of these concepts is beyond the scope of this review, readers should refer to the available resources that can be found elsewhere for additional background information as required $(1,2)$.

In this review, the most recent data on pertussis epidemiology in infectious disease textbooks and also in related scholarly articles has been reviewed. MeSH-term words including [pertussis] or [whooping cough] and [epidemiology] or [outbreak] or [resurgence] were our primary focus in screening the more recent data about the changing epidemiology of pertussis in Scopus, but our search was not restricted to this particular strategy; we also tried to find all of the most recent available data in the general field through other means as well.

\subsection{Introduction}

Bordetella pertussis is a small gram-negative respiratory pathogen, and it is the main etiologic agent of pertussis (whooping cough). Pertussis is a highly communicable disease with an attack rate of 100 percent in susceptible individuals. Pertussis infection is the cause of substantial morbidity around the world. It is estimated that 16 million cases of pertussis occur annually, and about 195,000 deaths occur per year (3).

Before the introduction of the pertussis vaccine, pertussis was a common cause of illness and death among younger infants and children. Typical pertussis has a prolonged course with three phases. The initial or catarrhal stage is characterized by upper respiratory symptoms that last for one to two weeks; the second or paroxysmal stage is marked by sudden attacks of severe cough with a characteristic "whoop" and post-tussive vomiting that lasts for 
several weeks. Finally, the third or convalescent stage is accompanied by gradual resolution of the coughing spells and lasts for several months. Pertussis is also one of the most important causes of chronic cough among adolescents and young adults due to the waning immunity in previously immunized individuals. Confirmation of clinically suspected cases of pertussis is based on culture or polymerase chain reactions (PCR) of a nasopharyngeal swab. However, more sensitivity has been found with the use of molecular tests (4).

Neither infection nor vaccination can provide permanent immunity, and despite the high vaccination coverage in childhood, the disease continues to be one of most the serious bacterial infections among vaccine preventable diseases (5-13).

Although there are improved surveillance systems, revised index case definitions and more accurate diagnostic tests may also have played a role in the recent increase in reports of the disease's incidence; nevertheless, a genuine increase in the incidence rate of the disease may itself have been a factor. Without national and local surveillance, it is difficult to analyze and interpret the epidemiological trends both between and within countries. Vaccination coverage and immunization schedules, case definition, diagnostic challenges, and alterations in vaccine types for routine immunization programs all may influence the major obstacles for control of this disease in different parts of the world.

\section{Evidence Acquisition}

This study attempts to investigate the barriers that exist against control of the disease in different regions of the world, and also provides current evidence for its prevention through the use of different strategies. Finally, the best recommendations will be proposed for disease control in Iran in light of considering the various strategies available.

\section{Results}

\subsection{Epidemiology}

The oldest reported epidemic of pertussis had been reported by Bahaodowle Razi, a Persian physician, in 15th century AD (14). Pertussis still continues to be an important public health problem in developing countries to this day (15). Despite the current prevention strategies, pertussis bouts occur with large multi-annual epidemics interspersed between cycles of a smaller amplitude (16).
Efforts to produce a vaccine with fewer side effects and a better safety profile were initially met with the introduction of the acellular pertussis vaccine. Although this vaccine has few adverse effects compared with the whole-cell vaccine, it has important effects on changing the epidemiology of pertussis (see discussion of vaccine effectiveness, vaccine type, and colonization rate in the following paragraphs).

The world health organization (WHO) continues to recommend whole-cell vaccines in resource-limited countries for primary pertussis vaccination (17). The latest WHO reports indicate that the most recent rates of infant pertussis-related deaths remained either similar to or lower than those of previous pertussis epidemics in the past two decades. Additionally, the latest reports of WHO's SAGE indicated a resurgence in disease-related morbidity and mortality in Chile, Portugal, the United States, and the United Kingdom in recent years (18). However, the exact mortality rates of pertussis in certain regions (as in Iran, for instance) have not been accurately determined, and the available reports may not be conclusive. There are also some conflicting interpretations of the data from certain reports (19).

A shorter duration of immunity after switching from a whole-cell pertussis vaccine to an acellular pertussis vaccine, the omission of the 18-month booster dose, and the low vaccine coverage outside of capital cities are considered to be the main factors that have led to the pertussis resurgence in younger children (two to three years of age). These findings are not universally observed in other countries using acellular vaccines. According to the SAGE report, the causes of the resurgence vary in different areas, including decreases in vaccine coverage in Chile, increased use of polymerase chain reaction testing (PCR) and disease reporting in Portugal, switching from whole-cell pertussis vaccines to acellular types, and the consequently shorter duration of protective immunity in the United States and the United Kingdom (18).

The current data have failed to support the idea that there may be a resurgence in Japan (one of the first countries that approved acellular vaccine use universally in 1981) and Thailand (which uses the whole-cell pertussis vaccine), while switching from the whole-cell vaccine to the acellular type was accompanied by an increase in pertussis incidences in 2006 in Singapore (18). Coverage of the primary pertussis vaccine series is acceptable in Iran as reported by WHO (99\%, 99\%, and 98\% for DTP1, DTP3, and DTP4, respectively) $(20,21)$. However, pertussis continues to occur in highly-immunized school-aged children (22, 23). After a decrease in the reported cases of whooping cough in 1990 in Iran, a slow increase was observed from 2000 to 2013. However, the number of reported cases has 
been decreasing since $2014(21,24)$. The available data reported by WHO are summarized in Figure 1. Characteristics of the natural cycle of pertussis epidemics in Iran remain unknown because of poor surveillance systems for reporting and registration. Although universal mandatory laboratory reporting is essential for surveillance of pertussis infection, a revision in the case definition has been recommended by some authors instead (25).

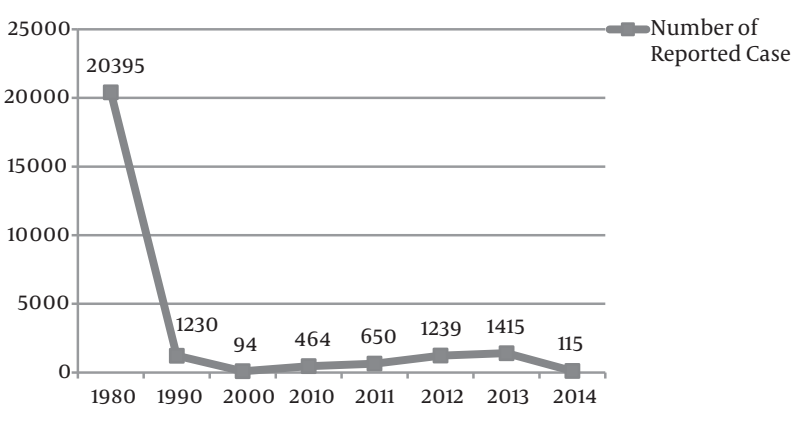

Figure 1. Frequency of Reported Cases of Pertussis in Iran After 1980 (Data Collected From "WHO 2015 Global Summary" (22))

PCR and the culture method are not routinely available for clinical case confirmation in Iran, so the outbreaks are difficult to precisely identify. Without knowing the accurate incidence rate of pertussis among different age groups and various geographic regions, any estimation for tracing epidemiologic changes remains difficult.

Furthermore, any decision to change the national immunization programs, including replacing new types of vaccines, adding or removing of certain vaccine booster doses, or introducing vaccines for certain groups should be based on new changes in pertussis epidemics and local population-based studies in each region. In the following paragraphs, we try to discuss some of the important factors that influence pertussis resurgence.

\subsubsection{Vaccine Effectiveness}

Decreases in vaccine effectiveness over time, especially with acellular vaccines, have been proposed as a possible cause of the resurgence. This fact has been addressed by researchers and confirmed by various observational studies during the disease epidemics (2, 26-28). Augmented T-helper-2 responses instead of T-helper-1 have also been suggested as an explanation for the waning antibody responses with these types of vaccines (29).

Serological response is not necessarily associated with disease protection (30). Nevertheless, preliminary studies have shown that the antibody response to acellular vaccines is equal to or even greater than that of whole-cell vaccines. More recent studies have revealed that the protection period of acellular vaccines is shorter than that of the whole-cell vaccines (31). The half-life of antibody resistance to the pertussis antigens following DTaP and DTP vaccination or natural infection is estimated to range from between six to 12 months and is not considerably different between either type of antigenic induction (30). The protection period of acellular vaccines is shorter than that of whole-cell vaccines. Some reports indicate that the decline in acellular vaccine effectiveness even after a year reaches 35 to 70 percent (28). It is estimated that children vaccinated with the DTaP vaccine are four to 15 times more at risk of acquiring pertussis years after the final dose of the primary immunization series in comparison to infants who receive the whole-cell vaccine (32). Waning immunity after the Tdap vaccine (as a booster dose) is even faster, with a reported 50\% reduction in its efficacy after two years (27).

A recent outbreak which occurred among preschoolaged children in Florida (USA) raised important concerns about the effectiveness of the acellular pertussis vaccine in children between the ages of one and five (33). Still, despite the variable vaccine effectiveness reported in children who were vaccinated with acellular vaccines, the development of pertussis infection outbreaks has also been shown in children vaccinated with whole-cell vaccines in certain regions including Iran (23).

\subsubsection{Pathogen Adaptation}

Antigenic alteration in vaccine strain and overproduction of the pertussis toxin are the proposed mechanisms for pathogen adaptation that may have contributed to the resurgence of pertussis. Long-lasting B-cell memory (cellmediated immune response) and the efficacy of antibodies (humoral response) are both affected by antigenic divergence (34). Certain proteins included in acellular vaccines are vulnerable to the mutation targets resulting in antigenic divergence. These proposed mutations could affect pertussis transmissibility. Furthermore, the efficacy of the antibodies may diminish after such antigenic changes.

Overproduction of the pertussis toxin may alter both innate and acquired immune responses. New alleles of the pertussis toxin promoters (ptxP3 strains) are responsible for pertussis toxin overproduction. In some regions, these new strains are replaced with previously circulating strains (35-37). In addition to mutations affecting pertussis toxin promoters that result in phenotypic changes in B. pertussis populations, obvious changes have also been detected in pertactin-type dominancy and tracheal colonization factor A (tcfA) in B. pertussis populations over time (37). Genes encoding pertactin (Prn) and the tracheal colonization factor have been suggested to be responsible for changing the ability of colonization. Strains containing 
Prn2 and Prn3 have a higher potency for colonization versus those that have Prn1, and a higher colonization rate leads to increased transmissibility (2). Some researchers have also proposed that pathogen adaptation (increasing strain fitness) correlates with accelerated waning of immunity by decreasing the period in which pertussis vaccines are effective (34).

Changes in the B. pertussis strain (such as pertactindeficient B. pertussis) are one of the other concerns regarding the efficacy of the currently-available acellular vaccines (38). Pertactin is a surface protein which plays an important role in the attachment of $B$. pertussis to the host cells. Recently, polymorphism in the pertactin (Prn) gene has been described as Prn1-3. Vaccine strains and circulating strains produce Prn1 and Prn2, respectively (39). Exposure to these novel strains puts those who are vaccinated with acellular type vaccines that contain pertactin as an antigenic surface protein at greater risk for pertussis. Pertactin-deficient $B$. pertussis strains have been reported in ten countries worldwide (40-44).

Pertactin-deficient $B$. pertussis strains do not express the DTaP antigen (Pertactin) and demonstrate more virulence than those vaccinated with the currently-available acellular vaccines such as DTaP and Tdap (45). Full pertussis vaccinees are 3.7 times more likely to be infected with pertactin-deficient strains (since they are at a selective advantage for loss of pertactin expression in B. pertussis) (32, 45).

These findings have shown better suitability of pertactin-deficient isolates in populations who have been administered these vaccines (46). On the whole, acellular pertussis vaccines are more effective in protecting individuals from disease development rather than preventing infection. A comparative effectiveness evaluation of acellular versus whole-cell vaccines has revealed that whole-cell type vaccinees are at lesser risk of disease progression after exposure to wild type pertussis $(17,26)$.

\subsubsection{Pertussis Vaccine Strain}

One additional point regarding genetic studies should be added to the previous discussion on vaccine effectiveness and bacterial adaptation. Recent findings have shown that the Tohama strain was no longer found in circulating isolates in the post vaccine-era (mainly in those countries that use whole-cell vaccines). These findings are important because the Tohama strain is currently the main strain applied for genomic sequencing in vaccine production (34) and a new generation of vaccines should be considered for solving this problem $(38,47)$.

In a recent study in Iran, strains in current vaccine and clinical isolates were reported to have low (approximately $40 \%$ ) similarities (48).

\subsubsection{Bordetella parapertussis}

Bordetella parapertussis can also cause a similar clinical syndrome. However, whooping cough due to B. parapertussis usually has a shorter duration (49). Virulence factors produced by $B$. parapertussis have some differences from those generated by B. pertussis, as the former does not express pertactin and fimbria proteins. B. parapertussis circulates independently of the pertussis vaccination without any changes in its prevalence or diagnostic characteristics $(49,50)$. There are different presumptions regarding the increase in the proportion of pertactin-deficient isolates (such as B. parapertussis) as a consequence of acellular pertussis vaccine usage; for instance, some researchers have proposed that acellular vaccine usage may be associated with increased susceptibility to $B$. parapertussis infection (51).

\subsubsection{Vaccine Type and Colonization Rate}

Based on animal studies, acellular vaccines are unable to prevent pertussis colonization. Thus, transmission may happen among vaccinees (52).

Recently, some questions regarding the efficacy of the currently-available whole-cell vaccines (in Iran and other parts of the world) have been raised by some researchers. This finding may be due to the heterogeneity of vaccine strains and the circulating clinical isolates, and can explain the occurrence of the disease in young children who are not expected to suffer from waning antibody responses $(48,53,54)$.

\subsubsection{Lack of Sufficient Booster Doses in Areas That Solely Use the Whole-Cell Vaccine}

In countries like Iran that use the whole-cell vaccine without additional adolescent booster doses, waning immunity is expected after six years from the last dose and, consequently, increased incidences of the disease occur among adolescents and young adults. This trend in pertussis prevalence among those of older ages may cause atypical clinical pictures (increased subclinical infection) with delayed diagnosis of pertussis in adults, resulting in increased transmissibility (55-57). In a recent multicenter study, the positive anti-pertussis toxin (IgG) (with a cutoff point of $94 \mathrm{U} / \mathrm{mL}$ ) was found in nearly one third (31.6\%) of freshman college students from different parts of Iran. The authors concluded that the high incidence of seropositivity is strong evidence for recent infection with $B$. pertussis, and that this population serves as a pertussis carrier, causing further circulation of the disease among high risk groups such as young infants (57). 
3.2. Lessons From Previous Epidemics and Predicted B. Pertussis Epidemics

Despite the previously discussed research, there is a lot of data about the changing epidemiology of pertussis. The resurgence of pertussis has been reported in both countries using mainly whole-cell vaccines as well as in those using mainly acellular pertussis vaccines $(18,32)$ and perhaps it can be said that the type of vaccination has not changed the frequency of pertussis epidemic cycles, which happen every three to five years.

One of the best designed reports for understanding these changes in the epidemiology of pertussis has been published by Pesco et al. (2) in 2014. In this survey, a different model for assessing vaccine effectiveness and effective transmission (contact) rates was designed and the possible outputs were provided and analyzed. These outputs were compared with real epidemiological changes in different states in the United States between 1993 and 2013. In this mathematical model, after inducing a linear decrease in vaccine effectiveness (VE) during a given period of time, a gradual increase in pertussis incidence was predicted. In contrast, changes in transmissibility that are defined by effective contact rates showed differences in comparison with reduction in VE. When these estimated models of the epidemics were compared with the rates for the real epidemics, interesting similarities were shown for each model. These models were therefore able to explain the possible underlying reason for the changing epidemiology and increase our knowledge about disease resurgence in different geographic regions (2).

Although such studies may provide predictable data about the changing epidemiology, it should be acknowledged that VE and effective transmission rates are directly or indirectly affected by other factors; for example, transmission rate may be affected by control measures taken during influenza epidemics annually. Also when decreases in VE are accompanied by changes in effective transmission rate, the final changes in disease epidemiology may not be predictable.

\subsection{Cost-Effectiveness of Switching to the Acellular Vaccine for the Iranian Population}

Because on the previously discussed reasons and the lack of adolescent booster Tdap doses in immunization programs in some parts of the world, including Iran, four strategies were recently analyzed and discussed in terms of their cost-effectiveness for the Iranian population by the pediatric infections research center and department of social medicine in Shahid Beheshti University of Medical Sciences. In the first theoretical strategy, the whole-cell vaccine was considered for immunization of all children and the acellular pertussis vaccine for immunization of highrisk groups; in the second strategy, immunization with the acellular pertussis vaccine was considered for all children in addition to those in high-risk groups; in the third strategy, the whole-cell vaccine was considered for immunization of all children and the acellular pertussis vaccine for immunization of high-risk groups with a booster dose for eligible adolescents and adults; finally, in the last strategy, the acellular pertussis vaccine was considered for immunization of all children without immunization of the other groups. These four scenarios were investigated using a decision tree model that was designed under the supervision of the Iranian ministry of health. Implementation of the pertussis immunization project with the acellular vaccine in the four default scenarios was quite cost effective, with cost effectiveness increases smaller than the per capita gross domestic product(GDP)(i.e., 4220 dollars). Based on this report, implementation of the first scenario with the highest level of cost effectiveness thought to be placed on the policy agenda (58).

\section{Conclusions}

Despite the high coverage of pertussis immunization among different age groups, pertussis infection continues to persist as a public health problem throughout the world, including in Iran. Many attempts have been made by researchers to determine why, despite this high vaccine coverage, that pertussis continues to circulate in epidemics in different parts of the world. According to the previously discussed aspects of pertussis epidemics, it seems that the occurrence of such outbreaks is multifactorial and based on VE, pathogen adaptation, changes in circulating pertussis strains, other pertussis species that are not contained in the current pertussis vaccines, vaccine type and colonization rate, and the lack of sufficient boosters. These factors all play important roles in the current increasing tendency for disease prevalence. Decreases in vaccine coverage have also influenced the resurgence of pertussis in young children in some parts of the world (18).

Among the main strategies proposed for the prevention of severe disease and related death in very young infants (including cocooning, immunization of adolescents and pregnant woman, and primary immunization of newborns), the cocoon strategy (vaccination of all adults in close contact with susceptible infants) showed diverse effects on the disease burden in countries applying cocooning immunization practices. Although decreases in infant ( $<6$ months) death had been reported after successful implementation of the cocoon strategy in Chile (since 2012), the overall disease incidence rate had not changed. Failure of acceptable coverage of this strategy in France was ac- 
companied with little benefits (18). This strategy involves timely vaccination of parents and caregivers, and therefore the overall impact, cost-effectiveness (economic issues), and acceptance by the family or caregivers are other important issues that affect the successful implementation of this strategy. Because of its limited success, it is not strongly recommended to adopt the cocoon strategy for protection against infant pertussis $(18,59,60)$.

Therefore, according to the high incidence rate of pertussis infection in older age groups in Iran (57, 61-66), additional booster doses for adolescents and also vaccination of pregnant women seems to be the preferred method to improve the current immunization program's efficacy against occurrences of pertussis infection. Revision to the traditional vaccine strain that is included in current vaccine production is also recommended.

Whole-cell pertussis vaccines (mainly DTP) are frequently accompanied by diverse adverse side effects. Although there are more side effects to account for with the whole-cell pertussis vaccine as compared to the acellular pertussis vaccine, we agree with the current recommendation by WHO that the primary vaccination practice with whole-cell vaccines be continued in conjunction with the implantation of acellular pertussis vaccine for revaccination in adolescents and adults (including pregnant women) via additional booster doses.

\section{Footnotes}

Authors' Contribution: Study concept and design, Iraj Sedighi; acquisition of data, Ali Amanati; drafting of the manuscript, Ali Amanati; critical revision of the manuscript for important intellectual content, Abdollah Karimi; final approval of the manuscript and agreement to be accountable for all aspects of the paper, Iraj Sedighi, Ali Amanati and Abdollah Karimi.

Financial Disclosure: The authors did not have any financial or other relationships which could be regarded as conflicts of interest.

\section{References}

1. Centers for Disease Control Prevention. Introduction to Epidemiology. Principles of epidemiology in public health practice: an introduction to applied epidemiology and biostatistics. Atlanta: Centers for Disease Control Prevention; 2006. p. 18.

2. Pesco P, Bergero P, Fabricius G, Hozbor D. Modelling the effect of changes in vaccine effectiveness and transmission contact rates on pertussis epidemiology. Epidemics. 2014;7:13-21. doi: 10.1016/j.epidem.2014.04.001. [PubMed: 24928665].

3. National Center for Immunization and Respiratory Diseases Pertussis (whooping cough), Fast Facts Centers for Disease Control and Prevention 2015. Available from: http://www.cdc.gov/pertussis/fast-facts. html.
4. Nikbin VS, Shahcheraghi F, Lotfi MN, Zahraei SM, Parzadeh M. Comparison of culture and real-time PCR for detection of Bordetella pertussis isolated from patients in Iran. Iran J Microbiol. 2013;5(3):209-14 [PubMed: 24475325].

5. Centers for DiseasePrevention. Pertussis epidemic-Washington, 2012 MMWR Morb Mortal Wkly Rep. 2012;61(28):517-22. [PubMed: 22810264].

6. Cherry JD. Epidemic pertussis in 2012-the resurgence of a vaccinepreventable disease. N Engl J Med. 2012;367(9):785-7. doi: 10.1056/NEJMp1209051. [PubMed: 22894554].

7. Fathima S, Ferrato C, Lee BE, Simmonds K, Yan L, Mukhi SN, et al. Bordetella pertussis in sporadic and outbreak settings in Alberta, Canada, July 2004-December 2012. BMC Infect Dis. 2014;14:48. doi: 10.1186/14712334-14-48. [PubMed: 24476570].

8. Matthias J, Dusek C, Pritchard SP, Rutledge L, Kinchen P, Lander M, et al. Notes from the field: Outbreak of pertussis in a school and religious community averse to health care and vaccinations-columbia County, Florida, 2013. MMWR Morb Mortal Wkly Rep. 2014;63(30):655. [PubMed: 25078655]

9. Miranda C, Wozniak A, Castillo C, Geoffroy E, Zumaran C, Porte L, et al. [Presence of Bordetella holmesii in an outbreak of pertussis in Chile]. Rev Chilena Infectol. 2013;30(3):237-43. doi: 10.4067/S071610182013000300001. [PubMed: 23877774].

10. Rodgers L, Martin SW, Cohn A, Budd J, Marcon M, Terranella A, et al. Epidemiologic and laboratory features of a large outbreak of pertussis-like illnesses associated with cocirculating Bordetella holmesii and Bordetella pertussis-Ohio, 2010-2011. Clin Infect Dis. 2013;56(3):322-31. doi: 10.1093/cid/cis888. [PubMed: 23087388].

11. Sizaire V, Garrido-Estepa M, Masa-Calles J. Increase of pertussis incidence in 2010 to 2012 after 12 years of low circulation in Spain. Euro Surveill. 2014;19(32).

12. Theofiles AG, Cunningham SA, Chia N, Jeraldo PR, Quest DJ, Mandrekar JN, et al. Pertussis outbreak, southeastern Minnesota, 2012. Mayo Clin Proc. 2014;89(10):1378-88. doi: 10.1016/j.mayocp.2014.08.004. [PubMed: 25282430].

13. Wu Y, Zhu B, Gao Y, Shi Z, Wang J, Wang H, et al. Clustered cases of Bordetella pertussis infection cause high levels of IgG antibodies against pertussis toxin in adolescents in Gaobeidian city, China. Epidemiol Infect. 2014;142(4):738-43. doi: 10.1017/S0950268813003099. [PubMed: 24308586].

14. Yarmohammadi H, Bahmani Kazeruni MH, Soofi A, Zargaran A. The First Report of Epidemic Pertussis by Bahaodowle Razi From the 15th Century Anno Domini. Iran Red Crescent Med J. 2015;17(7):13454. doi: 10.5812/ircmj.13454. [PubMed: 26413316].

15. Gentile A, Bhutta Z, Bravo L, Samy AG, Garcia RD, Hoosen A, et al Pediatric disease burden and vaccination recommendations: understanding local differences. Int J Infect Dis. 2010;14(8):649-58. doi: 10.1016/j.ijid.2009.11.006. [PubMed: 20181506].

16. Lavine JS, King AA, Andreasen V, Bjornstad ON. Immune boosting explains regime-shifts in prevaccine-era pertussis dynamics. PLoS One. 2013;8(8):72086. doi: 10.1371/journal.pone.0072086. [PubMed: 23991047].

17. World Health Organization. Revised guidance on the choice of pertussis vaccines: July 2014. Wkly Epidemiol Rec. 2014;25:337-40.

18. World Health Organization WHO SAGE pertussis working group Background paper. SAGE April World Health Organization; 2014. Available from: http://www.who.int/immunization/sage/meetings/2014/ april/1_Pertussis_background_FINAL4_web.pdf?ua=1.

19. Decker MD, Hosbach P, Johnson DR, Pool V, Greenberg DP. Estimating the effectiveness of tetanus-diphtheria-acellular pertussis vaccine.J Infect Dis. 2015;211(3):497-8. doi:10.1093/infdis/jiu477. [PubMed: 25156560].

20. World Health Organization Immunization, Vaccines and Biologicals Immunization surveillance, assessment and monitoring; Country Summaries of WHO/UNICEF estimated coverage World Health organization; 2014. Available from: http://www.who.int/immunization/ monitoring_surveillance/en/. 
21. World Health Organization WHO vaccine-preventable diseases: monitoring system 2015 global summary World Health Organization; 2015. Available from: http://apps.who.int/immunization_ monitoring/globalsummary/countries?countrycriteria[country][]= IRN\&commit=OK

22. Ghanaie RM, Karimi A, Sadeghi H, Esteghamati A, Fallah F, Armin SH, et al. Frequency of pertussis in iranian school-age children. J Pediatric Infect Dis. 2013;8(1):1-5.

23. Shahcheraghi F, Lotfi MN, Parzadeh M. Isolation of Bordetella pertussis and bordetella parapertussis from clinical specimens at different provinces of Iran in 2009-2010. J Mazandaran Univ Med Sci. 2012;22(88):1-8.

24. Shamshiri AR, Fahimzad A, Tabatabaie SA, Shiva F, Kadivar M, Khatami $A$, et al. Frequency of pediatric acute respiratory tract infections in Iran; A Systematic Review. Arch Pediatr Infect Dis. 2013;1(2):44-52.

25. Ghanaie RM, Karimi A, Sadeghi H, Esteghamti A, Falah F, Armin S, et al. Sensitivity and specificity of the World Health Organization pertussis clinical case definition. Int J Infect Dis. 2010;14(12):1072-5. doi: 10.1016/j.ijid.2010.07.005. [PubMed: 20951620].

26. Klein NP, Bartlett J, Fireman B, Rowhani-Rahbar A, Baxter R. Comparative effectiveness of acellular versus whole-cell pertussis vaccines in teenagers. Pediatrics. 2013;131(6):1716-22. doi: 10.1542/peds.2012-3836. [PubMed: 23690518].

27. Koepke R, Eickhoff JC, Ayele RA, Petit AB, Schauer SL, Hopfensperger DJ, et al. Estimating the effectiveness of tetanus-diphtheria-acellular pertussis vaccine (Tdap) for preventing pertussis: evidence of rapidly waning immunity and difference in effectiveness by Tdap brand. J Infect Dis. 2014;210(6):942-53. doi: 10.1093/infdis/jiu322. [PubMed: 24903664].

28. Acosta AM, DeBolt C, Tasslimi A, Lewis M, Stewart LK, Misegades LK, et al. Tdap vaccine effectiveness in adolescents during the 2012 Washington State pertussis epidemic. Pediatrics. 2015;135(6):981-9. doi: 10.1542/peds.2014-3358. [PubMed: 25941309].

29. Plotkin SA. Complex correlates of protection after vaccination. Clin Infect Dis. 2013;56(10):1458-65. doi: 10.1093/cid/cit048. [PubMed 23386629].

30. Berbers GA, van de Wetering MS, van Gageldonk PG, Schellekens JF, Versteegh FG, Teunis PF. A novel method for evaluating natural and vaccine induced serological responses to Bordetella pertussis antigens. Vaccine. 2013;31(36):3732-8. doi: 10.1016/j.vaccine.2013.05.073. [PubMed: 23742995].

31. McCarthy M. Acellular vaccines provided less protection during California pertussis outbreak. BMJ. 2013;346:3325. doi: 10.1136/bmj.f3325. [PubMed: 23694748].

32. Warfel JM, Edwards KM. Pertussis vaccines and the challenge of inducing durable immunity. Curr Opin Immunol. 2015;35:48-54. doi: 10.1016/j.coi.2015.05.008. [PubMed: 26091979].

33. Matthias J, Pritchard PS, Martin SW, Dusek C, Cathey E, D'Alessio R, et al. Sustained Transmission of Pertussis in Vaccinated, 1-5-Year-Old Children in a Preschool, Florida, USA. Emerg Infect Dis. 2016;22(2):2426. doi: 10.3201/eid2202.150325. [PubMed: 26814429].

34. Mooi FR, Van Der Maas NA, De Melker HE. Pertussis resurgence: waning immunity and pathogen adaptation - two sides of the same coin. Epidemiol Infect. 2014;142(4):685-94. doi: 10.1017/S0950268813000071. [PubMed: 23406868].

35. Mooi FR. Bordetella pertussis and vaccination: the persistence of a genetically monomorphic pathogen. Infect Genet Evol. 2010;10(1):36-49. doi:10.1016/j.meegid.2009.10.007. [PubMed: 19879977].

36. Advani A, Gustafsson L, Ahren C, Mooi FR, Hallander HO. Appearance of Fim3 and ptxP3-Bordetella pertussis strains, in two regions of Sweden with different vaccination programs. Vaccine. 2011;29(18):343842. doi: 10.1016/j.vaccine.2011.02.070. [PubMed: 21396900].

37. Petersen RF, Dalby T, Dragsted DM, Mooi F, Lambertsen L. Temporal trends in Bordetella pertussis populations, Denmark, 19492010. Emerg Infect Dis. 2012;18(5):767-74. doi: 10.3201/eid1805.110812. [PubMed: 22515990]
38. Hegerle N, Guiso N. Bordetella pertussis and pertactin-deficient clinical isolates: lessons for pertussis vaccines. Expert Rev Vaccines. 2014;13(9):1135-46. doi: 10.1586/14760584.2014.932254. [PubMed: 24953157].

39. He Q, Makinen J, Berbers G, Mooi FR, Viljanen MK, Arvilommi H, et al. Bordetella pertussis protein pertactin induces type-specific antibodies: one possible explanation for the emergence of antigenic variants?. J Infect Dis. 2003;187(8):1200-5. doi: 10.1086/368412. [PubMed: 12695998].

40. Pawloski LC, Queenan AM, Cassiday PK, Lynch AS, Harrison MJ, Shang W, et al. Prevalence and molecular characterization of pertactin-deficient Bordetella pertussis in the United States. Clin Vaccine Immunol. 2014;21(2):119-25. doi: 10.1128/CVI.00717-13. [PubMed: 24256623].

41. Queenan AM, Cassiday PK, Evangelista A. Pertactin-negative variants of Bordetella pertussis in the United States. $N$ Engl J Med. 2013;368(6):583-4. doi: 10.1056/NEJMc1209369. [PubMed: 23388024].

42. Lam C, Octavia S, Ricafort L, Sintchenko V, Gilbert GL, Wood $\mathrm{N}$, et al. Rapid increase in pertactin-deficient Bordetella pertussis isolates, Australia. Emerg Infect Dis. 2014;20(4):626-33. doi: 10.3201/eid2004.131478. [PubMed: 24655754].

43. Bamberger E, Abu Raya B, Cohen L, Golan-Shany O, Davidson S, Geffen Y, et al. Pertussis Resurgence Associated with Pertactin-Deficient and Genetically Divergent Bordetella Pertussis Isolates in Israel. Pediatr Infect Dis J. 2015;34(8):898-900. doi: 10.1097/INF.0000000000000753. [PubMed: 25966915].

44. Zeddeman A, van Gent M, Heuvelman CJ, van der Heide HG, Bart MJ, Advani A, et al. Investigations into the emergence of pertactindeficient Bordetella pertussis isolates in six European countries, 1996 to 2012. Euro Surveill. 2014;19(33) [PubMed: 25166348].

45. Martin SW, Pawloski L, Williams M, Weening K, DeBolt C, Qin X, et al. Pertactin-negative Bordetella pertussis strains: evidence for a possible selective advantage. Clin Infect Dis. 2015;60(2):223-7. doi: 10.1093/cid/ciu788. [PubMed: 25301209].

46. Bouchez V, Guiso N. Bordetella pertussis, B. parapertussis, vaccines and cycles of whooping cough. Pathog Dis. 2015;73(7) doi:10.1093/femspd/ftv055. [PubMed: 26242280].

47. Caro V, Bouchez V, Guiso N. Is the Sequenced Bordetella pertussis strain Tohama I representative of the species?. J Clin Microbiol. 2008;46(6):2125-8. doi:10.1128/JCM.02484-07. [PubMed: 18385436].

48. Haghighi F, Shahcheraghi F, Abbasi E, Eshraghi SS, Zeraati H, Mousavi SA, et al. Genetic Profile Variation in Vaccine Strains and Clinical Isolates of Bordetella pertussis Recovered from Iranian Patients. Avicenna J Med Biotechnol. 2014;6(3):178-84. [PubMed: 25215182].

49. Novotny P. Pathogenesis in Bordetella species. J Infect Dis. 1990;161(3):581-3. [PubMed: 2313135]

50. Johnson K. Recent Pertussis Uptick May Come From a Different Species. Medscape Medical News from the Interscience Conference of Antimicrobial Agents and Chemotherapy (ICAAC) 2015 2015. Available from: http://www.medscape.com/viewarticle/851371?nlid= 88731_2046\&src=wnl_edit_medn_peds\&uac $=195134 \mathrm{CV} \&$ spon $=$ 9\&impID $=839399 \& \mathrm{faf}=1$.

51. Long GH, Karanikas AT, Harvill ET, Read AF, Hudson PJ. Acellular pertussis vaccination facilitates Bordetella parapertussis infection in a rodent model of bordetellosis. Proc Biol Sci. 2010;277(1690):2017-25. doi: 10.1098/rspb.2010.0010. [PubMed: 20200027].

52. Warfel JM, Zimmerman LI, Merkel TJ. Acellular pertussis vaccines protect against disease but fail to prevent infection and transmission in a nonhuman primate model. Proc Natl Acad Sci U S A. 2014;111(2):787-92. doi: 10.1073/pnas.1314688110. [PubMed: 24277828].

53. Dashti AS, Karimi A, Arjmand R, Moghadami M, Kheirkhah T, Shiva F, et al. Serologic evidence of pertussis infection in vaccinated Iranian children. Iran J Med Sci. 2012;37(4):260-5. [PubMed: 23390332].

54. Zhang L, Prietsch SO, Axelsson I, Halperin SA. Acellular vaccines for preventing whooping cough in children. Cochrane Database Syst 
Rev. 2014(9):1478. doi: 10.1002/14651858.CD001478.pub6. [PubMed: 25228233].

55. Sedighi I, Sadrosadat T. Acellular Pertussis Vaccine: Iran's Next Step to Control the Disease?. Arch Pediatr Infect Dis. 2015;3(2):23918.

56. Sedighi I, Sadrosadat T. Comment on: Seroprevalence of Bordetella pertussis antibody in pregnant women in Iran.J Res Health Sci. 2014;14(3):236-7. [PubMed: 25209914].

57. Sedighi I, Rahimi H, Emadoleslami MS, Fahimzad A, Hosseini F, Afsharian M, et al. Seroepidemiology of bordetella pertussis infection in fresh college students in Iran: a multicenter study. Arch Pediatr Infect Dis. 2014;9(1)

58. Ghanaie MR, Karimi A, Moradi M, Yaghobi M, Zahraee M, Mahmodi S. Acellular pertussis vaccine cost-effectiveness analysis in Iran. Vaccination in Special Satuation. Tehran: Pediatric Infection Research Center; 2014. p. 64.

59. Wiley KE, Zuo Y, Macartney KK, McIntyre PB. Sources of pertussis infection in young infants: a review of key evidence informing targeting of the cocoon strategy. Vaccine. 2013;31(4):618-25. doi: 10.1016/j.vaccine.2012.11.052. [PubMed: 23200883].

60. Castagnini LA, Healy CM, Rench MA, Wootton SH, Munoz FM, Baker CJ. Impact of maternal postpartum tetanus and diphtheria toxoids and acellular pertussis immunization on infant pertussis infection. Clin Infect Dis. 2012;54(1):78-84. doi: 10.1093/cid/cir765. [PubMed:
22075790]

61. Hashemi SH, Zamani M, Mamani M, Javedanpoor R, Rahighi AH, Nadi E. Seroprevalence of Bordetella pertussis antibody in pregnant women in Iran. J Res Health Sci. 2014;14(2):128-31. [PubMed: 24728747]

62. Hashemi SH, Ranjbar M, Hajilooi M, Seif-Rabiei MA, Bolandi M, Moghimi J. Seroprevalence of Immunoglobulin $\mathrm{G}$ antibodies against pertussis toxin among asymptomatic medical students in the west of Iran: a cross sectional study. BMC Infect Dis. 2009;9:58. doi: 10.1186/1471-2334-9-58. [PubMed: 19426546].

63. Eslamifar A, Ramezani A, Banifazl M, Khadem-Sadegh A, Aghakhani A Seroprevalence of IgG Antibodies against Bordetella pertussis in Different Age Groups in Tehran, Iran. Iran J Pathol. 2012;7(3):171-6.

64. Hajia M, Rahbar M, Fallah F, Safadel N. Detection of Bordetella pertussis in Infants Suspected to have Whooping Cough. Open Respir Med J. 2012;6:34-6. doi: 10.2174/1874306401206010034. [PubMed: 22754598].

65. Sedaghat M, Nakhost Lotfi M, Talebi M, Saifi M, Pourshafie MR. Status of pertussis in iran. Jundishapur J Microbiol. 2014;7(11):12421. doi: 10.5812/jjm.12421. [PubMed: 25774274].

66. Shojaei J, Saffar M, Hashemi A, Ghorbani G, Rezai M, Shahmohammadi S. Clinical and laboratory features of pertussis in hospitalized infants with confirmed versus probable pertussis cases. Ann Med Health Sci Res. 2014;4(6):910-4. doi: 10.4103/2141-9248.144911. [PubMed 25506485]. 